\title{
ESTRATEGIAS DE MUJERES FERROVIARIAS FRENTE A LAS VIOLENCIAS DE GÉNERO. UNA EXPERIENCIA DESDE LA PSICOLOGÍA SOCIAL COMUNITARIA
}

\author{
María Malena Lenta \\ Roxana Gabriela Longo \\ Graciela Zaldúa \\ Universidad de Buenos Aires, Argentina
}

\begin{abstract}
RESUMEN
La segregación ocupacional, el acoso y la discriminación son modos en que las violencias de género se expresan en el ámbito trabajo, siguiendo patrones del orden patriarcal. El ferrocarril es un espacio laboral tradicionalmente masculino. La emergencia de mujeres allí visibiliza modalidades de subalternización social de este colectivo. Desde el enfoque de psicología social comunitaria, estudios de género y un proceso de Investigación Acción Participativa, nos propusimos analizar la participación social y las estrategias frente a las violencias de género de mujeres ferroviarias. Implementamos talleres, grupos de discusión, relatos de vida y observaciones naturalísticas con una muestra intencional de 65 participantes. Entre los resultados se identificó el reconocimiento de las violencias internas y externas al espacio laboral y transformaciones subjetivas y colectivas mediadas por el proceso de participación social.
\end{abstract}

\section{Palabras clave}

Género, investigación acción participativa, trabajadoras

\begin{abstract}
Occupational segregation, harassment and discrimination are ways in which gender violence is expressed in the workplace, following patterns of the patriarchal order. The railway is a traditionally male workplace. The emergence of women there makes visible modalities of social subalternization of this group. From the focus of community social psychology, gender studies and a Participatory Action Research process, we developed the objective of analyzing social participation and strategies against gender violence of railway women. We implemented workshops, discussion groups, life stories and naturalistic observations with an intentional sample of 65 participants. Among the results was identified the recognition of internal and external violence to the workplace and subjective and collective transformations mediated by the process of social participation.
\end{abstract}

Keywords

Gender, participatory action research, women workers

Correspondence about this article should be addressed to María Malena Lenta.Email: MalenaLenta@gmail.com 


\section{STRATEGIES OF WOMEN RAILWAY AGAINST GENDER VIOLENCES. AN EXPERIENCE FROM COMMUNITY SOCIAL PSYCHOLOGY}

La prevención de las violencias y, en particular, de las violencias de género constituye un componente central de las demandas actuales de la sociedad civil, en particular, del movimiento feminista y de las identidades disidentes. Interpela al campo de las políticas sociales y a las representaciones y prácticas cristalizadas que reproducen desigualdades en las diversas instituciones sociales y ámbitos de la vida cotidiana.

Para Zizek (2008), la violencia, en tanto exceso de agresividad o plus de dominio sobre la/el otra/o, actúa sincrónicamente en tres modalidades: la violencia intersubjetiva, la violencia simbólica y la violencia sistémica. La violencia intersubjetiva opera en el plano interpersonal con la modalidad psicológica y/o física. Es un tipo de violencia visible y sancionable en la sociedad, sin embargo, es tan solo la punta del iceberg de los complejos órdenes de producción y reproducción social de las violencias y, a menudo, funciona omitiendo dicha totalidad compleja que incluye a las otras dos formas de la violencia: la sistémica y la simbólica. La violencia sistémica es estructurante del sistema capitalista y los regímenes de opresión como el patriarcado y el racismo. La violencia simbólica alude a las ideologías y los discursos que enuncian al otra/o como inferior y permiten legitimar las violencias intersubjetivas a la vez que justifican la violencia sistémica. Ambas -la sistémica y la simbólica- son violencias objetivas, pero se encuentran invisibilizadas.

La violencia hacia las mujeres que es definida en Argentina por la Ley nacional 26485 de Erradicación contra todas las formas de violencia hacia las mujeres en todos los ámbitos en los que desarrollen sus relaciones interpersonales, como toda conducta, acto u omisión basada en el poder desigual de los géneros que, de manera directa o indirecta, afecte el cuerpo, la sexualidad, el patrimonio y hasta la vida de una mujer o persona con identidad disidente, en cualquier ámbito en donde se desarrollen sus relaciones interpersonales, debe ser comprendida en estos tres niveles señalados por Zizek y no puede ser reducida a su vertiente visible (Longo, Lenta y Zaldúa, 2018). La violencia hacia las mujeres, aprehendida entonces como violencia de género, se instala desde el régimen patriarcal que ordena culturas, costumbres, moral y normalidad entre los cuerpos sexuales generizados y desigualados a través de discursos e ideologías de género, que hacen a que los episodios de violencia interpersonales no solo no sean excepcionales, sino que incluso pueden ser naturalizados o banalizados.

La identificación de la violencia contra las mujeres como un problema de poder, es decir, producto de un sistema estructural de opresión patriarcal, indica que la violencia de género no es un problema individual, privado, familiar, sino un problema público y social estrechamente vinculado con los derechos y la ciudadanía. Esta mirada remite a la distribución social de los derechos y deberes, de las cargas y los beneficios, donde las mujeres y las identidades disidentes, se encuentran en una situación de negación de sus derechos (Sagot, 2008). En este marco, la exigibilidad de derechos y de la justiciabilidad de género permite analizar las rutas críticas por las que transitan las personas y colectivos violentados, así como también, recuperar los proyectos de emancipación que promueven cambios legales, culturales y sociales (Riquelme y Barrientos, 2014).

La discriminación, la vulnerabilidad ante los abusos, los acosos y los asedios que viven las mujeres y las personas con identidades disidentes plantean problemáticas emergentes de la violencia de género en el campo del trabajo. En el imaginario social se presume que las discriminaciones por razón de género están despareciendo en todos los ámbitos de la esfera pública y, sin embargo, las monitorizaciones estadísticas, del balance entre mujeres y varones, siguen confirmando notables desigualdades en relación con la tasa de actividad, la brecha salarial o la segregación ocupacional (Barrancos, 2014).

Según la Organización Internacional del Trabajo (OIT) (2016), a escala global, el 52,1 por ciento de las mujeres y el 51,2 por ciento de los hombres en el mercado de trabajo son trabajadores asalariados y a sueldo. No obstante, esto no implica de por sí una garantía de trabajo de calidad. De hecho, a escala mundial, casi el 40 por ciento de las mujeres con empleos remunerados no contribuyen a la protección social, es decir, se encuentran precarizadas, frente al 27 por ciento de los varones. A su vez las mujeres continúan estando excesivamente representadas (en comparación con su porcentaje en el empleo total) en dos grupos ocupacionales: "Trabajadores administrativos, de los servicios y del comercio" y "Ocupaciones 
elementales", lo cual implica al 50 por ciento de los sectores peor remunerado. Y también las mujeres continúan estando excesivamente representadas como trabajadoras familiares auxiliares alcanzando al 20 por ciento de las mismas, frente al 8,1 por ciento de los varones. Al mismo tiempo, las mujeres se encargan al menos dos veces y media más en labores de cuidado y limpieza que los varones, implicando que trabajen jornadas más largas que los varones cuando se toma en consideración tanto el trabajo remunerado como no remunerado.

A las transformaciones en las relaciones del trabajo con la precarización y flexibilización laboral se añade la emergencia del acoso laboral habitualmente soslayado o negado. El acoso laboral en el espacio del trabajo es un problema que afecta a todas las personas. Sin embargo, según la OIT (2017) quienes cuentan con menores cuotas de poder en el espacio laboral, son quienes enfrentan mayor desprotección frente al acoso. Este es el caso, por ejemplo, de las mujeres quienes son acosadas sexualmente por sus superiores jerárquicos o pares, asediadas por su condición de madres o situación de embarazo y discriminadas para el acceso a determinados puestos y categorías laborales por su condición de género. Frente a la ilusión de igualdad, no solo se observa el fenómeno de "techo de cristal" sino también, el de "suelo pegajoso" que señala cómo las mujeres son segregadas ocupacionalmente a partir de una división sexual del trabajo que las ubica no solo en tareas esterotipadas de género (cuidado, asistencia, limpieza, etc) sino también desvalorizadas tanto en el plano del poder (categorías inferiores) como de la remuneración.

En el sector ferroviario, históricamente construido como una profesión masculina, estas segregaciones persisten a nivel de fenómeno internacional. Shirley Burman (1994, 2009) y Helena Wojtczak (2005), resaltan la interesada invisibilización de la contribución de las mujeres al sector ferroviario. El ferrocarril desde sus orígenes hasta el presente ha limitado el acceso de las mujeres al empleo. No sólo ofrece pocas oportunidades de empleo a las mujeres, sino que su contratación se polariza en ocupaciones tipificadas como "femeninas" porque estos bastiones de cuasi-exclusividad masculina siguen asociándose con imaginarios de virilidad, es decir, como puestos que requieren fuerza, en ocupaciones de demostrada penosidad y para las que a menudo se prefiere a candidatos con dotes de mando (Martin y Arnard, 2013).

Ballesteros (2013) describe cómo las mujeres han ingresado históricamente al espacio del trabajo ferroviario en calidad de "hijas" o "esposas" de trabajadores del sector a cumplir tareas poco calificadas como las de guardabarreras o aprendices, lo que insiste en su lugar subalternizado de género. Sin embargo, en la última década, la participación social y política de las mujeres frente a las violencias de género interpela el lugar de las propias mujeres en los diferentes ámbitos en donde desarrollan sus relaciones interpersonales incluyendo, al ámbito del trabajo (Bonacorsi y Carrario, 2012). En este marco, al abordar la experiencia de las mujeres trabajadoras ferroviarias contemporáneas en Argentina, surgen algunos interrogantes: ¿Cómo opera la participación social de las mujeres en la producción de estrategias contra las violencias de género en el espacio de trabajo? ¿Qué violencias se visibilizan en el trabajo a partir de la participación social de las mujeres? ¿Qué transformaciones subjetivas y colectivas se identifican?

\section{Sobre la Participación Social de las Mujeres}

En el contexto latinoamericano, los aportes de la psicología social comunitaria han sido destacados en cuanto al propósito de aportar a la transformación de las condiciones de opresión, segregación y desventaja de diferentes sectores de la comunidad (Wiesenfeld, 2016). Para Montero (2010b), su objeto de estudio está constituido por los procesos psicosociales que organizan la vida cotidiana de los sujetos, tanto aquellos vinculados a la reproducción social (habituación, familiarización y naturalización) como los que se vinculan con la transformación social (desnaturalización, concientización y desideologización) y que permiten desarrollar, fomentar y mantener el control y poder que los sujetos y los colectivos pueden ejercer sobre sus condiciones materiales de existencia. De esta manera, los procesos de investigación-acción desencadenados por la psicología social comunitaria instan a la desideologización de la vida cotidiana a partir de la desnaturalización de las condiciones de opresión y subordinación de género, clase, etnia y 
generación; a la historización de los procesos colectivos y a la potenciación de los recursos de la propia comunidad o colectivo (Parker, 2010; Dobles, 2015).

Por ello, las dinámicas de la participación comunitaria constituyen un aspecto central para comprender la praxis de la psicología social comunitaria. Para Montero (2010a), la participación debe ser analizada en los territorios no solo en relación con los tipos y modalidades que se observan entre los agentes internos (personas interesadas y grupos organizados dentro de las comunidades) y los otros agentes externos participantes (las/los profesionales, técnicos y otros actores que desarrollan prácticas con grupos y comunidades), sino que también debe considerarse el tipo de compromiso en la participación.

Comprender la relación bilateral entre participación y compromiso permite evidenciar dos aspectos de un mismo fenómeno: la participación comprometida y el compromiso participativo. La participacióncompromiso implica un proceso complejo, colectivo, libre e incluyente; con variedad de actores, de acciones y de niveles. Se trata de una relación generadora de relaciones que se orienta por valores y objetivos compartidos, cuya realización supone transformaciones singulares y colectivas. Asimismo, supone un proceso que permite producir la transformación recíproca (entre el sujeto o sujetos y el objeto), la autorrealización y es una condición para la libertad.

El compromiso-participativo implica a la conciencia y al sentimiento de responsabilidad y obligación respecto del trabajo y objetivos de un colectivo, comunidad, proyecto o causa, que conduce a acompañar, actuar y responder por las acciones desarrolladas, es decir, que compele a participar.

En las últimas décadas la masiva participación social de las mujeres en los diferentes movimientos sociales propone la reflexión sobre la configuración, las dinámicas, las necesidades y las prácticas de los sujetos involucrados en el proceso, puesto que enhebra nuevos desafíos sociales, políticos y culturales al calor de luchas emancipatorias. Las mujeres desafían, en muchos casos sin saberlo, un mandato social impuesto que intentan desarmar desde su accionar en la cotidianeidad de las organizaciones sociales (Zaldúa, Sopransi y Longo, 2007). Desde diferentes iniciativas y proyectos, la participación social de las mujeres genera procesos que, desde un enfoque innovador, se proponen incidir en la consecución de la equidad de género en distintos contextos. Son experiencias y acciones concretas que promueven cambios que contribuyen al fortalecimiento de una democracia de género, la autonomía personal y el empoderamiento colectivo, al interpelar las estructuras patriarcales que imponen modos desiguales de producir y valorar los cuerpos y las identidades en la sociedad (Ciriza, 2007).

\section{Estrategia Metodológica}

Desde un paradigma de investigación social cualitativa y crítica, este trabajo delimita al problema de estudio en un entramado histórico-social que es moldeado por valores y prácticas sociales, económicas, culturales, étnicas y de género. La metodología propuesta considera que todo proceso de producción de conocimiento es una construcción teórica que surge a partir de la acción desde una dialéctica de subjetivación y de objetivación de la realidad que se busca conocer. La creatividad, la reflexividad y el compromiso asumido con la realidad estudiada son aspectos clave de este enfoque investigativo (Minayo, 2009).

En consistencia con esta mirada, la apelación a la estrategia de la Investigación Acción Participativa (IAP) en este estudio intenta contribuir en la autonomía de pensamiento y acción de los colectivos sociales con los que se emprenden procesos de investigación. La IAP interroga a las prácticas del campo psicosocial y propone una praxis crítica, dialógica, participativa, reflexiva y problematizadora de las situaciones de desigualdad, opresión y las violencias. Asimismo, se espera que del proceso surjan dispositivos innovadores y socializadores de técnicas, propósito que contempla la reflexividad sobre los objetivos para investigar, intervenir y validar contextualmente (Zaldúa, Longo, Sopransi y Lenta, 2014).

Desde este marco y en función del problema planteado, se desarrolló un diseño de investigación de tipo exploratorio-descriptivo que permitió desarrollar el objetivo de: analizar la participación social y las estrategias frente a las violencias de género, desde la experiencia de un colectivo de trabajadoras ferroviarias, entre septiembre de 2016 y junio de 2017. A partir de una lógica cualitativa se intentó, mediante las narrativas de las participantes, interpretar las dinámicas de las prácticas y los significados de las historias que reconstruyen las mujeres sobre su vida cotidiana en relación al trabajo. En este sentido, experiencia, vivencia, sentido común, acción social, intencionalidad y significado constituyen la superficie 
en la cual se alza la posibilidad de la comprensión, actitud que es al mismo tiempo arte y ciencia de la investigación cualitativa (Minayo, 2010).

Participantes y muestra. En el estudio participaron 65 mujeres trabajadoras del Ferrocarril Sarmiento que contaban con contrato estable en el ferrocarril con fecha anterior al 31 de diciembre de 2015. La antigüedad en el ferrocarril presentó un rango de entre 11 años y 1 año al momento del estudio, identificándose la moda en 1,5 años de antigüedad. En cuanto al puesto de trabajo, las trabajadoras se desempeñaban en: limpieza, evasión, guardabarrera, guarda de tren, señalamiento y boletería. Respecto de la participación sindical, si bien todas las trabajadoras habían concurrido a al menos una actividad sindical (asamblea, reunión, paro, entre otras) desde su ingreso al ferrocarril, seis de ellas tenían cargos sindicales (cuatro de ellas eran miembros de la Comisión Ejecutiva de la Seccional Ferroviaria Gran Buenos Aires Oeste y dos eran miembras del Cuerpo de Delegados y la Comisión de Reclamos del sector).

En cuanto al rango de edad, el mismo fue de entre 20 años y 54 años. La moda se ubicó en los 31 años de edad. En cuanto a la situación de pareja, el 64,61 por ciento tenía pareja estable mientras que el 35,39 por ciento, no lo tenía. A su vez, el 80 por ciento tenía al menos un hijo/a, mientras que el 20 por ciento restantes no tenía hijos/as.

Tipo de muestreo. El tipo de muestreo fue intencional no probabilístico. Los casos se seleccionaron a partir de los criterios de accesibilidad y voluntariedad en la participación. Se buscó que la muestra fuera homogénea en cuanto a la participación en al menos una actividad sindical y heterogénea en cuanto al puesto de trabajo. El tamaño de la muestra estuvo sujeto a criterios de saturación conceptual.

Fuentes e instrumentos. Een un primer momento de indagación se trabajó a partir de la implementación de seis talleres de discusión sobre las problemáticas relevantes de las mujeres trabajadoras vinculadas a las violencias de género y el proceso de trabajo. Se trató de la creación de un espacio colectivo de identificación de problemas, consensos, disensos, nudos críticos y recursos colectivos (Kamberelis y Dimitriadis, 2015), en los que participaron las 65 trabajadoras ferroviarias.

En un segundo momento, se implementaron 10 relatos de vida a un conjunto de trabajadoras que habían participado de la primera instancia de talleres de discusión. A partir del desarrollo de entrevistas biográficas (Chase, 2015), se indagaron los siguientes tópicos propuestos por las investigadoras: trayectoria laboral anterior al ferrocarril, características de las relaciones afectivas y vinculares a partir del ingreso al ferrocarril, modalidades de participación sindical y de género y afectaciones subjetivas y colectivas de la participación. Se buscó producir datos de mayor intensidad a partir de la producción de narrativas singulares sobre la experiencia colectiva.

A su vez, durante todo el proceso se desarrolló una observación naturalística. Se trata de una perspectiva en donde la observación se asume desde una posición de-colonial respecto de las prácticas de investigación. A partir de la reflexividad de las investigadoras respecto de las determinaciones de clase, género, etnicidad y generación, entre otras, se busca superar la tensión entre observación y participación (Angrosino, 2015). Particularmente, en este trabajo se observaron y registraron en el cuaderno de campo reuniones de trabajo de las ferroviarias, asambleas, movilizaciones, espacios de formación y capacitación, acciones comunitarias, entre otras. La observación se focalizó en las características y las modalidades de gestión colectiva de las actividades de justicia de género desarrolladas por el colectivo.

Sistematización e interpretación de los datos: a partir de la desgrabación de las entrevistas y talleres, así como del tipeo del cuaderno de campo, se procedió a la organización de los mismos según las dimensiones de análisis de la investigación y de categorías analíticas emergentes de los datos, con el apoyo del software informático Atlas-ti versión 6.0. El trabajo de análisis priorizó una exégesis transversal de las narrativas de las participantes y registros observacionales, por sobre la exégesis singular, en función del problema de estudio. 


\section{Contextualización del escenario de investigación}

Como señalamos en estudios anteriores (Lenta, Longo y Zaldúa, 2018), el gremio ferroviario ha sido considerado históricamente como un sector fuertemente masculino. Con la conformación del sistema ferroviario argentino a mediados del siglo XIX como parte de la consolidación del estado-nación y el modelo agroexportador, el ferrocarril fue un medio de transporte privilegiado que permitió conectar económica y socialmente a casi la totalidad del nacional. En su apogeo alcanzó $47.000 \mathrm{~km}$ de vías. A mediados del siglo $\mathrm{XX}$, pasó en pocas décadas a vivir cambios bruscos.

Durante el primer gobierno peronista (1946-1952) los ferrocarriles fueron nacionalizados. Pero a los pocos años, a partir del gobierno de Frondizi (1958-1962), se inició un período de reorganización productiva y el retroceso ferroviario comenzó con el Plan Larkin (asesorado por el Banco Mundial) que redujo a la mitad la cantidad de vías. En ese período, el gobierno de Frondizi aplicó el código de justicia militar a través del Plan de Conmoción Interna del Estado (CONINTES) y muchos ferroviarios que resistieron a los cierres de ramales fueron reprimidos. Durante la última dictadura militar (1976-1983), caracterizada por la represión estatal genocida hacia las organizaciones y militantes sociales, el ajuste económico y la desindustrialización del país, la resistencia de los trabajadores ferroviarios fue un hito histórico en la defensa de sus fuentes de trabajo y el ferrocarril. No obstante, junto con los ramales y talleres, muchos trabajares fueron desaparecidos por el terrorismo de Estado.

Durante la década de 1990 con la presidencia de Menem (1989-1995/1995-1999) tuvo lugar el denominado "ferrocidio". Con la reprivatización del ferrocarril, no solo se cerraron ramales; sino que se clausuraron talleres, se echaron a perder maquinarias y la población ferroviaria disminuyó drásticamente con el despido de más de 135.000 trabajadores a comienzos de esa década (Díscoli, 2013; Cena, 2009). En los años posteriores continuó la decadencia ferroviaria con nuevas privatizaciones hasta que los trenes fueron nuevamente nacionalizados luego de la denominada "masacre de Once" en 2012, el accidente que causó la muerte de 53 pasajeros y cientos de heridos producto de las malas condiciones de seguridad que habían sido reiteradamente denunciadas por la Comisión de Reclamos de los trabajadores de la Línea Sarmiento (Zaldúa, Lenta y Leale, 2016).

En el contexto de la crisis del 2001, la debacle en la representación política tuvo un impacto en el modelo sindical y dio lugar a un nuevo sindicalismo combativo cuyo principio es la democracia sindical de base. Dentro de los diferentes procesos de organización que se desarrollaron en esa primera década del siglo XXI, uno de los casos más relevantes fue el de la seccional Gran Buenos Aires Oeste de la Unión Ferroviaria (Ferrocarril Sarmiento). Además de conseguir sustanciales mejoras salariales en esos primeros años, este sector del sindicalismo logró el armado de una bolsa de trabajo para el ingreso al ferrocarril donde cada uno de los trabajadores podrían proponer el ingreso de familiares (Castillo, 2012). Entre los años 2005 y 2006 en ese marco se dio el ingreso de las primeras 16 mujeres, actualmente, denominadas las "pioneras". Posteriormente, fueron ingresando nuevos grupos de mujeres hasta llegar, en 2016 al número de 300 mujeres y cerca de 3000 varones.

Como señalábamos en Lenta, Longo y Zaldúa (2018), el sindicato, el club ferroviario y la familia ferroviaria, conforman territorios en los que históricamente se socializaba una identidad común del personal ferroviario. Pero la presencia de mujeres en el espacio de trabajo, interpeló dicho proceso identitario, la distribución de roles y espacios, y dio lugar a nuevas discusiones al interior del sindicato y los sectores de trabajo.

Desde su ingreso, las mujeres ferroviarias llevaron adelante diferentes desafíos para habitar los espacios de trabajo y organización sindical. La primera exigencia colectiva de cupo femenino en todas las especialidades y las llevó a organizar la agrupación de trabajadoras ferroviarias "Mujer bonita es la que lucha". Desde este espacio de organización sindical y de género, participaron en espacios del movimiento

2 Cabe señalar que, previamente, a fines de los años de 1990, se había dado el ingreso de dos mujeres a causa del fallecimiento de sus maridos ferroviarios. Sin embargo, estos hechos no son consignados en la historización del proceso de las mujeres ferroviarias del Ferrocarril Sarmiento. 
de mujeres por el reconocimiento de sus derechos como los Encuentros Nacionales de Mujeres, entre otros, lo que configuró la necesidad de responder al problema de las violencias de género que vivían las trabajadoras en los diferentes ámbitos de la vida cotidiana, además de en el espacio laboral. En el año 2016, las 65 mujeres ferroviarias participantes de este trabajo, se capacitaron en la prevención de las violencias de género, a partir de un espacio implementado en co-gestión con el Observatorio de Prevención y Promoción de la Salud Comunitaria de la Facultad de Psicología de la Universidad de Buenos Aires, lo que constituyó el inicio del proceso de este IAP.

En un encuentro realizado en torno al 8 de marzo, día internacional de las mujeres trabajadoras en las que se nos convocó para trabajar sobre las problemáticas actuales de las trabajadoras, se hizo visible una situación de violencia de género en el ámbito doméstico vivida por una de las trabajadoras, a la cual las participantes querían acompañar. En el proceso de delinear las estrategias de acompañamiento del caso puntual y la exigibilidad de acceso a recursos a las políticas públicas, comenzó a elaborarse la propuesta de trabajo de IAP que implicó un primer momento de indagación de las problemáticas prevalentes visibilizadas por las trabajadoras como violencias de género, un segundo momento de capacitación mediante la elaboración del programa de "Formación de promotoras para la prevención de las violencias de género" y un tercer momento de trabajo de acompañamiento de casos que, en 2017, junto con un equipo de profesionales, permitió la creación de un dispositivo de acompañamiento a mujeres ferroviarias en situación de violencia de género denominado "La Casa que Abraza". Desde este espacio, además de recibir consultas sobre situaciones puntuales de violencias de género, se desarrollaron talleres de sensibilización del tema con varones y mujeres en el espacio de trabajo y se desarrollaron acciones de exigibilidad de derecho a la empresa ferroviaria como la demanda de la licencia por violencia de género y la formalización de un protocolo ante las situaciones de violencia de género en el espacio del trabajo, entre otras demandas. A su vez, durante el proceso, se trabajó con relatos de vida de las participantes del espacio de IAP y se organizaron diferentes grupos de discusión y problematización de los procesos desarrollados de manera conjunta.

\section{Resultados y Discusiones}

$\mathrm{El}$ abordaje de las narrativas de las trabajadoras ferroviarias en torno a los momentos y procesos de participación tanto en los espacios sindicales y como en los espacios de organización frente a las problemáticas específicas de género, permitió elaborar tres dimensiones de análisis emergentes de los datos y validados en los espacios dialógicos con las participantes del estudio: la visibilización de las violencias de género a partir de la participación social de las mujeres trabajadoras; las condiciones para la participación social de las mujeres y las transformaciones subjetivas; y, las estrategias colectivas frente a las violencias de género.

\section{La visibilización de las violencias de género a partir de la participación social de las mujeres trabajadoras}

La Convención sobre la eliminación de todas las formas de discriminación contra la mujer (CEDAW), en tanto normativa internacional pionera en 1979 en la visibilización de la violencia de género, estableció tempranamente que la expresión "discriminación contra la mujer" denota a toda distinción, exclusión o restricción basada en el sexo que tenga por objeto o resultado menoscabar o anular el reconocimiento, goce o ejercicio de los derechos de las mujeres en cualquier ámbito de la vida. Sin embargo, varias décadas después en el espacio ferroviario, las mujeres que trabajan en él están sujetas a altos niveles de discriminaciones por parte de la empresa o los compañeros varones, a través de expresiones discriminatorias, abusos de poder, pero también aspecto edilicios y materiales de trabajo. Dichas discriminación fueron identificadas como tales en los talleres de discusión sobre las violencias de género:

"Digamos, el hombre puede estar en cualquier lado, en la mujer la excusa era "no ustedes no van a poder levantar barrera, no tiene baño" como que era un problema 
si estábamos en ese lugar. Estas 13 chicas que (...) soportamos todo” (Susana, guarda de tren)

"Cuando nosotras ingresamos como no eran muchas las mujeres, en ese entonces había ropas masculinas, talles grandes. Había que arreglarse” (Marta, limpieza)

"Hubo bastantes problemas porque compartían los vestuarios. El tema del vestuario es el baño, la ducha. Vos te tenés que estar bañando y están los tipos y no hay más nadie entonces era un problema” (Ana, limpieza)

“(...) Un tema es la complicidad o entre los compañeros también. Vos como que quedas expuesta y tenés que aprender a manejarte con los mismos códigos y aprender a llevártela" (Nayla, taller)

Estas situaciones, que afectan de manera considerable el bienestar de las mujeres en el trabajo, se conjugan con una serie de repercusiones subjetivas en aquellas mujeres que acceden a puestos históricamente asumidos por varones.

En las interacciones cotidianas se presentan núcleos argumentativos legitimadores que apelaban a la debilidad física de la fisiología femenina y a la moralidad vinculada a los deberes familiares como principales motivos para justificar y legitimar la existencia de una normativa especial o de prácticas laborales que, de facto, operan como mecanismos discriminatorios (Espuny, 2008) y que circulan a través de mensajes, chistes o uso sistemático de lenguaje sexista, constituyendo 'micromachismos'.

Asimismo, las trabajadoras del transporte ferroviario relataron en los espacios de discusión colectiva que experimentan otras situaciones de violencia promovidas por terceros. Este tipo de violencia se suele denominar "violencia externa" y comprende, por lo general, a los insultos, las amenazas o la agresión física o psicológica ejercidos contra un trabajador/a por personas ajenas a la organización en la que trabaja, incluidos los usuarios y que ponen en peligro la salud, la seguridad o el bienestar del/la trabajador/a. Pero adquiere un carácter particular cuando se trata de trabajadoras mujeres:

“(...) también corres riesgos una vuelta un chabón me empezó a tirar piedras y por un tiempo estuve (...) media paranoica, porque no es sencillo" (Sol, banderillera) “(...) te quieren pasar por arriba acá: la empresa, los supervisores, los pasajeros, el público, los automovilistas, todos te quieren pasar por arriba porque piensan que porque sos mujeres, vos no podés. No estás capacitada con la fuerza (Marina, banderillera)

“(...) los pasajeros mismo abrían las puestas y te tiraban botellas, te tiraban piedras incluso algunos te hacían pis tenías que tener cuidado hasta de eso, de los autos, del tren y todo. Lo hacen como una manera de humillarnos porque somos mujeres" (Laura, guarda de tren)

"A nosotras nos dicen otra que cosa que a un varón. Le dicen 'dale pelotudo levanta la barrera' y a nosotras es toda una opinión sobre nuestro cuerpo, por qué estamos ahí paradas, sobre que no sabemos hacer la tarea, la de 'andá a lavar los platos' es lo mínimo, ya que ni te lo dicen" (Lina, banderillera)

La violencia vivida entre pares o con personas ajenas al espacio de trabajo, a pesar del malestar que produce, implica para ellas un menor costo personal y subjetivo porque en el proceso de organización de las mujeres como colectivo, han desplegado diversas experiencias de desnaturalización, problematización y solución de situaciones discriminatorias vividas en principio como un problema individual:

"Yo pensaba que era mi culpa que o algo que me pasaba a mí que un compañero fuera denso, pesado conmigo, que me invitara a salir todo el tiempo, aunque no daba (...) empecé a encontrarme con mis compañeras en otros espacios, en las reuniones, en los encuentros y me empecé a dar cuenta de que les pasaba a varias. Que no era mi culpa" (Brenda, guarda de tren y delegada) 
"Te vas dando cuenta al hablar con las compañeras que no son ellas las enemigas, sino que necesitamos ser más solidarias entre nosotras para poder enfrentar las cosas que nos pasan. No son problemas de cada una. A casi todas nos pasan casi las mismas cosas desde chistes hasta que no te dejen entrar a trabajar a ciertos puesto" (Sol, banderillera)

"Cuando te insulta un pasajero por el hecho de ser mujeres, claro que te da bronca. Pero también te podés llegar a reír... 'Pobre tipo que me manda a cocinar o a lavar los platos. Debe estar resentido con su vida y le molesta que las mujeres salgamos adelante" "(Carolina, evasión)

En el trascurso de su devenir laboral, las trabajadoras ferroviarias fueron saldando y afrontando diversas situaciones a través de espacios de reflexión de las situaciones vividas entre pares y de iniciativas de exigibilidad de derechos laborales equitativos en las que se establecieron diferentes medidas preventivas y de abordaje de las situaciones de violencia que favorecieron la morigeración de los sentimientos de culpa, la baja autoestima y el miedo, al historizar y contextualizar las violencias percibidas, así como también, al organizar acciones preventivas y/o reparatorias de las violencias vividas.

\section{Las condiciones para la participación social de las mujeres y las transformaciones subjetivas}

La experiencia de las mujeres trabajadoras ferroviarias evidencia el proceso de participación social que fueron construyendo como colectivo de mujeres. Frente a situaciones adversas o de discriminación laboral fueron buscando soluciones y construyeron iniciativas innovadoras que facilitaron su posición dentro del espacio de trabajo. Desde su activismo, las mujeres contribuyeron a discutir las prerrogativas masculinas y a enriquecer el debate sobre los derechos de las mujeres en el ámbito laboral. Interpelaron la racionalidad organizativa dentro del sector ferroviario, pero también problematizaron elementos de identidad colectiva y corporativa propia de la tradición ferroviaria (Ballesteros, 2013). Conquistaron diversos derechos: la integración de mujeres en ocupaciones tipificadas como masculinas, ubicaron el tema de la violencia de género y el acoso sexual como una cuestión relevante que las atraviesa en el ámbito laboral y cotidiano, desarrollaron campañas y promovieron buenas prácticas para abordar los problemas. Como colectivo de mujeres promovieron también instancias de capacitación o problematización de tipo mixtas.

En ese proceso de participación, las trabajadoras pudieron construir hitos colectivos y personales que delimitaron una identidad colectiva:

"Tuvimos una primera reunión (...) Y ahí nos empezó a hacer un poco de ruido por qué en el ferrocarril tenemos compañeras en limpieza en su gran mayoría, evasión y la que llegaban a boletería era como listo ya está termino tu carrera ahí “ (Marina, limpieza y delegada)

"Creo que lo primero que me recuerdo que más marcado fue cuando fuimos a Retiro a hacer un pedido formal de las "mujeres a la conducción" de trenes. Todavía no se logró , pero me parece que ese es el día que más recuerdo juntarnos todas e ir" (Verónica, telecomunicaciones)

"(...) creo que fue un espacio que conquistamos las chicas es muy importante, teniendo en cuenta que la línea siempre fue masculina. Esto de tener un espacio con el que podamos contar y que podamos hacer valer nuestros derechos, tener asesoría jurídica, psicológica porque no solamente la violencia es intrafamiliar de la compañeras, sino también laboral. Esto de plantearlo, de ver cómo afrontarlo porque los compañeros la mayoría como que está acostumbrado al ambiente masculino o de repente en sectores como los jefes de tren que son muy machistas" (Clara, limpieza) 
La posibilidad de establecer ciertos hitos -como las primeras reuniones de mujeres ferroviarias-, la enunciación de reclamos colectivos -como "mujeres a la conducción", exigencia del derecho a poder presentarse al examen para ser conductoras de trenes- junto con los logros visualizados -como la creación de "La Casa que Abraza" -, permiten configurar una identidad colectiva: "nosotras". Se trata de una identidad que no elude diferencias ni tensiones pero que se instala desde la sororidad en tanto reconocimiento de la otra como semejante y no, como competencia. A su vez estos espacios de participación promovieron repensar las historias personales y reconfigurar la identidad singular de cada una de ellas:

"Al principio me daba culpa cambiar de horario porque iba a dejar solos a mis hijos desde muy temprano o porque los dejaba para ir a una reunión. Ahora no, pienso que son decisiones que tengo que tomar por el trabajo, por el futuro mío y de ellos. A los hombres eso no les da culpa ¿por qué tendría que pasarme a mí?” (Brenda, guarda de tren y delegada)

"Cuando fui al Encuentro de Mujeres, fue la primera vez que me fui de viaje desde que tengo a mi nene. Fue muy fuerte, Si no hubiera sido por las chicas, no podría haberlo hecho. Me pude permitir ir sin culpas" (Amanda, telecomunicaciones) "Nos fuimos de vacaciones tres compañeras y yo. Nos fuimos yo sin los hijos y ellas, que son más jovencitas, que no tienen, pero sin los novios. Lo veo como parte de la organización que logramos" (Lía, banderillera)

La posibilidad de pensarse como mujeres más allá del sintagma mujer=madre impuesto desde los imaginarios e ideologías de género patriarcales; así como también transformar los afectos y proyectos de vida, surge de la experiencia de habitar otros espacios sociales tradicionalmente vedados para las mujeres. Este modo de participación social de las mujeres permite repensar sus haceres cotidianos en el ámbito laboral, pero también problematizar las experiencias a lo largo de su vida, fomentando la autonomía y la afirmación subjetiva.

\section{Las estrategias colectivas frente a las violencias de género}

Existen diversos aspectos que resaltan en la experiencia participativa emprendida por este colectivo de mujeres trabajadoras ferroviarias. Pero un aspecto a destacar es el desarrollo de estrategias innovadoras y colectivas que han desarrollado a lo largo de su proceso organizativo como colectivo. El espacio de "La Casa que Abraza" es una experiencia singular innovadora porque combina la acción de justicia de género con la organización sindical democrática con claras demandas vinculadas al mejoramiento de las condiciones y medioambiente de trabajo (Britwum y Ledwith, 2014). Durante 2017, las trabajadoras participantes de este espacio no solo han identificado y acompañado casos de violencia de género, sino que han logrado el reconocimiento por parte de la empresa de la "licencia por violencia de género" con el objetivo de despatologizar las violencias vividas por las mujeres para considerarlas como acciones relacionadas con el ejercicio del poder patriarcal. Asimismo, los espacios de formación mixtos para deconstruir los mitos sobre las violencias de género y visibilizar las formas naturalizadas de las violencias en el espacio de trabajo no solo han sido reclamados como instancias de capacitación obligatorias hacia el conjunto de las/os trabajadores del ferrocarril, sino que han sido puestas en práctica por las propias trabajadoras cuando la empresa no las garantizaba.

Estas acciones junto con otros logros han permitido construir una agenda demandas de equidad de género que se imbrica con otros reclamos comunes del espacio de trabajo y que solo son posibles a partir de la participación comprometida de las trabajadoras ferroviarias.

\section{Conclusiones}

La creación de espacios autogestivos de género y la implementación de estrategias de prevención de violencias de género, propician procesos instituyentes singulares y colectivos de reconocimiento sororo. La dinámica participativa sindical inscripta en "Mujer bonita es la que lucha" opera con una lógica de afirmación reivindicativa de género y habilita la producción de sentidos que subvierten los tradicionales 
estereotipos femeninos centrados en la esencialización de lo femenino en el sintagma mujer-madre y la subalternización del trabajo femenino en lo público.

La "La Casa que Abraza" constituye un dispositivo instituyente de subjetividades más autónomas y habilita espacios de prevención de las violencias y desde una sensibilidad y responsabilidad ética-política.

Las cuestiones de género, en particular las lógicas de poder y subordinación, son obstáculos en la constitución de relaciones más igualitarias entre mujeres, varones e identidades disidentes. Las mujeres son víctimas más frecuentes de la violencia de género en el trabajo, a través de procesos de segregación, hostigamiento y/o discriminación laboral. Es un hecho que se vive en la cotidianeidad y que se expresa tanto en la organización de las jerarquías laborales, las capacidades y accesibilidades y en las relaciones intra e entre los géneros en el ambiente laboral.

Repensar el proceso de IAP permitió dar cuenta del fortalecimiento del propio colectivo de las mujeres trabajadoras que progresivamente fueron visibilizando logros y transformaciones en el plano personal y en el de la organización colectiva al poder historizar su lugar y participación en el territorio del ferrocarril y vincularlo su posición de subalternidad social en tanto mujeres.

Las instancias de historización y problematización de la vida cotidiana de las mujeres en el espacio del trabajo, pero también del hogar, favorecieron también la posibilidad de identificar temas prioritarios a abordar y elaborar para ello estrategias para reorganizar su posición social al proponerse ocupar otros espacios sociales y al permitir-se abandonar otros vinculados a los mandatos sociales de género.

El ejercicio reflexivo que se fue desarrollando en las instancias participativas entre las mujeres que hicieron parte activa del proceso de IAP, pero también en otras en las que se trabajó con compañeros varones o con compañeras con menor participación comprometida en la IAP, también favoreció visibilizar que la libertad sindical, el derecho de sindicalización y la negociación colectiva constituyen una base central para avanzar sobre el derecho a la igualdad y no discriminación de género.

La prevención de las violencias de género es un campo que reclama experiencias creativas e integrales. Junto con la necesidad de innovar en los abordajes y prácticas, resulta un insumo fundamental del campo de la psicología social comunitaria sistematizar experiencias exitosas promovidas desde la participación social de los sujetos subalternizados, en este caso, las mujeres trabajadoras.

En tanto praxis implicada en la promoción del fortalecimiento comunitario y en la transformación de las situaciones de injusticia e inequidades de género, la psicología social comunitaria tiene los desafíos de:

- Promover procesos de gestión asociada entre la universidad pública y los colectivos de mujeres trabajadoras para la co-construcción de conocimientos frente a las modalidades de las violencias que viven las trabajadoras y el desarrollo de estrategias innovadoras para su abordaje.

- Visibilizar las experiencias de participación social de las mujeres trabajadoras en la lucha contra las violencias de género, a través de tecnologías sociales para su difusión.

- Implementar procesos de transferencia de experiencias y herramientas construidas hacia otros colectivos de mujeres trabajadoras. 


\section{Referencias}

Angrosino, M. (2015). Recontextualización de la observación. En T. Denzin, \& Y. Lincoln, Manual de investigación cualitativa. Volumen IV. Métodos de recolección y análisis de datos (págs. 203-234). Buenos Aires, Argentina: Gedisa.

Ballesteros, E. (2013). Las mujeres en el ferrocarril: Acceso restringido. En Actas de XI Congreso Nacional de Sociología. Visitado el 15/6/2018 en: http://fes-sociologia.com/files/congress/11/papers/122.pdf

Barrancos, D. (2014). Géneros y sexualidades disidentes en la Argentina: de la agencia por derechos a la legislación positiva. En Cuadernos Intercambio sobre Centroamérica y el Caribe, Vol. 11, No. 2 Julio-Diciembre, 2014, 17-46

Bonacorsi, N. y Carrario, M. (2012). Participación de las mujeres en el mundo sindical. Un cambio cultural en el nuevo siglo. En La Alijaba, 16 (2), 125:140.

Britwum, A. y Ledwith, S. (2014). Labor and globalization. Mering, Alemania: Rainer Hampp Verlag.

Burman, S. (1994). Women and the American Railroad - Documentary Photography. En Journal of the West 21(2), 36-41.

Burman, S. (2009). Women and Railroading. En Trains Railroad History 16, 15-22.

Castillo, Ch. (2012). El sindicalismo combativo bajo el nuevo gobierno de Cristina Fernández de Kirchner. En Memorias de las Jornadas de Sociología de la UNLP. Visitado el 11/03/2017 en http://www.memoria.fahce.unlp.edu .ar/library?a=d\&c=eventos\&d=Jev1780

Cena, J. C. (2009). El ferrocidio. Buenos Aires, Argentina: La nave de los locos.

Chase, S. (2015). Investigación narrativa. En T. Denzin, \& I. Lincoln, Manual de investigación cualitativa. Volumen IV. Métodos de recolección y análisis de datos (págs. 58-112). Buenos Aires, Argentina: Gedisa.

Ciriza, A. (2007). “¿En qué sentido se dice ciudadanía de mujeres? Sobre las paradojas de la abstracción del cuerpo real y el derecho a decidir“. En Hoyos Vázquez, G. (Comp.) Filosofía y teorías políticas entre la crítica y la utopía. Buenos Aires, Argentina: CLACSO.

Díscoli, J. I. (2015). Memoria e identidad de los trabajadores ferroviarios; el riel y el acero como carnadura viva de un proyecto histórico soberano, en XIII Jornadas Rosarinas de Antropología Socio-Cultural. Visitado el $11 / 5 / 2018$ en http://rephip.unr.edu.ar/bitstream/handle/2133/5440/discoli.pdf?sequence=3\&isAllowed=y

Dobles, I. (2015). Psicología de la liberación y psicología comunitaria latinoamericana. Una perspectiva, en Teoría y Crítica de la Psicología 6, 122:139.

Kamberelis, G., y Dimitriadis, G. (2015). Grupos Focales. En T. Denzin, \& Y. Lincoln, Manual de investigación cualitativa. Volumen IV. Métodos de recolección y análisis de datos (págs. 494-452). Buenos Aires, Argentina: Gedisa.

Lenta, M.; Longo, R. y Zaldúa, G. (2018). "Trabajadoras ferroviarias. Del mundo masculino a La Casa que Abraza”. En Zaldúa, G., Longo, R., Lenta, M. y Bottinelli, M. (comps.) Dispositivos instituyentes sobre géneros y violencias (págs. 27-44). Buenos Aires, Argentina: Teseo.

Longo, R., Lenta, M. y Zaldúa, G. (2018)."Dispositivos de prevención y asistencia frente a las violencias de género“. En Zaldúa, G., Longo, R., Lenta, M. y Bottinelli, M. (comps.) Dispositivos instituyentes sobre géneros y violencias (págs. 45-62). Buenos Aires, Argentina: Teseo.

Martin, P. y Arnard, A. (2013). The experience of women in male-dominated occupations: a constructivist grounded theory inquirí. En Journal of Industrial Psychology, 39(2), 31-43.

Minayo, M. (2009). La artesanía de la investigación cualitativa. Buenos Aires, Argentina: Lugar.

Minayo, M. (2010). Los conceptos estructurantes de la investigación cualitativa. En Revista Salud Colectiva 6(3), 251:261.

Minayo, M. (2010). Los conceptos estructurantes de la investigación cualitativa. En Revista Salud Colectiva 6(3), 251:261.

Ministerio de Trabajo de la Nación (2014) Boletín de Estadísticas de Género y Mercado de Trabajo. Buenos Aires, Argentina: Ministerio de Trabajo de la Nación. 
Ministerio de Trabajo de la Nación (2016) Boletín de Estadísticas de Género y Mercado de Trabajo. Buenos Aires, Argentina: Ministerio de Trabajo de la Nación.

Montero, M. (2010a). Fortalecimiento de la Ciudadanía y Transformación Social: Área de Encuentro entre la Psicología. En Psyhé (19)2, 51:63.

Montero, M. (2010b). Teoría y práctica de la Psicología Comunitaria: la tensión entre comunidad y sociedad. Buenos Aires, Argentina: Paidós.

Organización Internacional del Trabajo (2016). Informe: las mujeres en el trabajo. Ginebra, Suiza: Organización Internacional del Trabajo.

Organización Internacional del Trabajo (2017) Acoso laboral hacia las mujeres. Ginebra, Suiza: Organización Internacional del Trabajo. Visitado el 11/5/2018, en: https://www.ilo.org/wcmsp5/groups/public/---americas/---ro-lima/---srosan_jose/documents/publication/wcms_220029.pdf

Parker, I. (2010). La psicología como ideología: contra la disciplina. Madrid, Estado Español: La catarata.

Riquelme, M.C. y Barrientos, A. (2014). Políticas públicas para la igualdad de género: un aporte a la autonomía de las mujeres. Libros de América Latina y el Caribe (CEPAL).

Sagot, M. (2008). Ruta crítica de las mujeres afectadas por la violencia intrafamiliar en américa latina (estudios de caso de diez países). Lima, Perú: Organización Panamericana de la Salud.

Wiesenfeld, E. (2016). Trascendiendo confines disciplinares: continuidad, psicología comunitaria crítica y psicología social comunitaria, al revés. En Interamerican Journal of Psychology 50(1), 4-13.

Wojtctzak, H. (2009). Railwaywomen. Exploitation. Betrayal and triumph in the workplaces. London, UK: Hastings.

Zaldúa, G, Sopransi, M. y Longo, R. (2007). Vulnerabilidad, género y prácticas de autonomía en dos organizaciones de trabajadores desocupados en Gral. Mosconi y Conurbano Bonaerense. En Anuario de investigaciones (14)2, 183:198.

Zaldúa, G., Lenta, M. y Leale, H. (2016). "Los trabajadores ferroviarios y el efecto traumático del desastre de Once”. En Zaldúa (Org.) Intervenciones en psicología comunitaria (págs. 345-351). Buenos Aires, Argentina: Teseo.

Zaldúa, G.; Longo, R.; Lenta, M. y Sopransi, M. (2014). Exigibilidad de derechos de personas en situación de prostitución y dispositivos comunitarios en CABA, en Anuario de Investigaciones (19)2, 1-23.

Zizek, S. (2013). Sobre la violencia. Seis reflexiones marginales. Buenos Aires, Argentina: Paidós. 